\title{
Irreversibility of Heat Conduction in Complex Multiphase Systems and Its Application to the Effective Thermal Conductivity of Porous Media
}

\author{
Qun Chen ${ }^{1}$, Moran Wang ${ }^{2}$, Ning Pan*,4, Zeng-yuan Guo ${ }^{1}$ \\ 1 Department of Engineering Mechanics, Tsinghua University, Beijing 100084, China \\ 2 Los Alamos National Laboratory, Los Alamos, NM 87545, USA \\ 3 Department of Biological \& Agricultural Engineering, University of California, Davis, CA \\ 95616, USA \\ 4 Center of Physics of Fibrous Soft Matters, Donghua University, Shanghai 200051, China
}

\begin{abstract}
The irreversibility of heat conduction in porous media, its relation to effective thermal conductivities (ETCs), and the optimization of thermal conduction process are investigated in this work based on the concept of entransy dissipation. Two more new concepts of reference entransy dissipation and nondimensional entransy dissipation are first introduced. Then it is showed that the nondimensional entransy dissipation rate (NER) can be employed as an objective function to evaluate the efficiency of a thermal transfer process in a porous material. By using this criterion and a newly developed structure growth algorithms, different porous structures were generated and the corresponding values of both ETC and NER were derived to illustrate the usefulness and power of using NER to assess the thermal performance of the materials.

The results show that the effective thermal conductivity not only influences the heat transfer ability of porous media, but also reflects the irreversibility of heat conduction in porous media, which is a dissipation coefficient for heat transfer. Meanwhile, decreasing the structural particle size will increase the contact points, i.e. more heat bridges, decrease the temperature gradient nearby the contact points, and hence significantly increase the effective thermal coefficient of porous media. Essentially, decreasing the particle size will result in a more uniform distribution of both temperature gradient and local entransy dissipation rate along the heat flow direction, and consequently lead to a larger effective thermal conductivity.
\end{abstract}

Keywords: irreversibility; entransy dissipation; effective thermal conductivity; particle size; porous media

\section{Introduction}

The effective thermal conductivity (ETC) is one of the most important properties of complex media, for its significance in such wide areas as engineering design, energy saving, geophysical exploration, biological and medical engineering ${ }^{[1-8]}$, and has been of great interests for scientific research and investigation. With respect to the design function, which is the goal of engineering

Corresponding author. Email: ningpan.pan@gmail.com disciplines ${ }^{[9]}$, optimizing the material ETC is often the key for efficiency and economy.

In most previous theoretical analyses, the macro material ETC was assumed a simple function of the phase fractions of the constitutes based on the effective medium theory (EMT) or the network combinations of Series and Parallel models [10-13], while the effects of phase distribution and inter-phase interactions were mostly ignored, leading to gross errors in prediction of the overall material ETC. Meanwhile, giving the difficulties encountered in seeking theoretical solutions on one hand, and tedious and intricate experimental measurements on the other, numerical approach has been 
regarded as a better alternative in dealing with behaviors of multiphase media, owing to the rapid development of computational techniques.

The first main step of effective numerical modeling of a complex medium has to include describing the structural details and the interactions in the material properly and adequately. Several structural reproduction methods have been proposed, including the discrete reduced distance method by Tacher et al. [14], the Monte Carlo annealing algorithm by Mohanty [15] and most recently the multi-parameter random generation-growth method, termed quartet structure generation set (QSGS) by Wang et al. ${ }^{[16]}$. The second step is to solve the governing equations efficiently by such available numerical tools as the traditional partial differential equation (PDE) solvers, including the finite difference ${ }^{[17]}$ and finite element methods ${ }^{[18]}$, the Monte Carlo method ${ }^{[19]}$ and the lattice Boltzmann method $[16,20]$. However, when the porous structure becomes complicated, especially when the multi-phase conjugate heat transfer problem is considered, the traditional PDE solvers not only demand huge or often prohibitive computational resources [21, 22], but often fail to obtain acceptable results as well. Therefore, in dealing with heat transfer processes in porous media with less grid number for the same accuracy, a high-efficiency lattice Boltzmann algorithm proposed by Wang et al. ${ }^{[16]}$ to solve the energy equations in multiphase transport problems becomes highly desirable; the method has shown to lead to predictions agree well with the theoretical results for some benchmark cases, and with the existing experimental date for much more complex multiphase porous media.

Based on the above theoretical, experimental or numerical methods, the researchers have derived a great deal of useful conclusions on porous media. For example, porosity is the most important factor that determines the ETC of porous media ${ }^{[23]}$, decreasing the average particle size increases the overall ETC, and enlarging the directional growth probability along the temperature gradient also enhances the ETC in this direction ${ }^{[16]}$. However, these conclusions are still of phenomenological descriptions, while the physical essentials lay deep underneath, which calls for further studies to explore the more fundamental issues.

As well known, heat transfer is an irreversible process. When heat transfers in an isothermal system from a higher temperature to a lower one, although the total quantity of the thermal energy is conserved, the same amount of energy is unable to cause a reverse of such heat flow without additional external energy. In other words, the heat transfer ability of the same energy is reduced by the "inherent equivalent thermal resistance". To characterize such heat transfer ability, a new physical concept, $\left(Q_{v} T\right) / 2$, termed as the entransy was introduced by Guo et al. ${ }^{[24]}$. Based on this new theory, the entransy dissipation was used to measure the irreversibility of heat transfer processes, and all of these concepts have been validated with numerous examples in heat conduction ${ }^{[24]}$, heat convection ${ }^{[25-27]}$ and heat radiation ${ }^{[28]}$

The objective of this contribution is to investigate the relationship between the ETC of porous media and the irreversibility of heat conduction processes in terms of the entransy dissipation. A few new concepts including the characteristic entransy dissipation and the nondimensional entransy dissipation (NER) are first introduced; and then the relationship between the NER and the ETC of multiphase media will be investigated for materials with regular and random microstructures, respectively. Finally, the particle size effects on the ETC of micro-granular porous media are further explained using the entransy dissipation analysis.

\section{Irreversibility of heat conduction in porous media}

For heat conduction in media without a heat source, the thermal energy conservation equation is

$$
\rho c_{p} \frac{\partial T}{\partial t}=-\nabla \cdot \dot{q}_{h},
$$

where $\rho$ is the density, $c_{p}$ the specific heat capacity, $T$ the temperature, $t$ the time, $\dot{q}_{h}$ the heat flux density.

Multiplying both sides of equation (1) by 
temperature, $T$, yields the entransy balance equation

$$
\rho c_{p} T \frac{\partial T}{\partial t}=-\nabla \cdot\left(\dot{q}_{h} T\right)+\dot{q}_{h} \nabla T .
$$

Introducing the entransy density $\varepsilon_{v h}=\frac{1}{2} \rho c_{p} T^{2}$, the balance equation (2) can be rewritten into

$$
\frac{\partial \varepsilon_{v h}}{\partial t}=-\nabla \cdot\left(\dot{\varepsilon}_{h}\right)-\phi_{h}
$$

The left term in equation (3), $\frac{\partial \varepsilon_{v h}}{\partial t}=\dot{\varepsilon}_{v h}$, is the time derivative of the internal entransy density. The first term on the right is the entransy transferred in the process, while the second term is defines as the dissipation function of entransy [24].

$$
\phi_{h}=-\dot{q}_{h} \cdot \nabla T=k|\nabla T|^{2} .
$$

Where $k$ is the thermal conductivity. Equation (4) resembles the dissipation function of mechanical energy in viscous fluid flow and can thus be termed as dissipation rate of the entransy. Integrating equation (4) in the entire computational domain gives the total entransy dissipation rate, which represents the overall irreversibility of heat conduction in the media.

$$
\Phi_{h}=\iiint_{\Omega} \phi_{h} d V=\iiint_{\Omega} k|\nabla T|^{2} d V .
$$

Where $\Omega$ is the heat conduction domain and $V$ is the total volume.

It is to note that unlike the thermal conductivity $k$ of a simple monolithic solid, the ETC for a complex multiphase system, $k_{\text {eff }}$, is no longer an intrinsic material property, for it is also related to such external factors as the thermal and geometry boundary conditions and the material internal structures including porosity. To assess the system behavior, we have to establish an objective function. Among the various ETCs the system can possess, we select the minimum one, $k_{e c}$, as the reference heat conductivity. The reference entransy dissipation rate, $\Phi_{h c}$, is then introduced as

$$
\Phi_{h c}=k_{e c}(\Delta T / L)^{2} V_{0} .
$$

where, $\Delta T$ and $L$ are the temperature difference and the distance between the hot end and the cold end, respectively. $V_{o}$ is the unit volume of the media. For a specific heat conduction process in a given multiphase system, $k_{e c}, \Delta T, L$ and $V_{\mathrm{o}}$ are all constant, so this reference entransy dissipation is a fixed value.

Furthermore, by scaling the local thermal conductivity $k$ with $k_{e c}$, the local temperature gradient with $\Delta T / L$ and the total volume with $V_{o}$, the non-dimensional entransy dissipation (NER) rate can be defined as

$$
N_{\phi h}=\iiint_{\Omega} \frac{k}{k_{e c}}\left(\frac{|\nabla T|}{\Delta T / L}\right)^{2} \frac{d V}{V_{o}}=\frac{\Phi_{h}}{\Phi_{h c}} .
$$

This NER rate, $N_{\phi h}$, is the objective function we intended to establish and it has the following characteristics:

a. $N_{\phi h} \geq 1$, thus representing the ratio of the entransy dissipation enhancement due to the presence of the phase with higher thermal conductivity. In practice for a multiphase system, $k_{e c}$ is the smallest one out of the thermal conductivities of all phases.

b. Unlike $k_{\text {eff }}$ (ETC), the system $N_{\phi h}$ (NER rate) is independent of other non-intrinsic conditions. $N_{\phi h}$ measures the heat transfer irreversibility, just like the electrical energy dissipation in electricity or mechanical energy dissipation in mechanics. In other words, NER describes the efficiency of a thermal conduction process in a material, whereas ETC reflect the material resistance to thermal transfer.

c. As a result, $N_{\phi h}$ can be used as a criterion, via variational calculus, to optimize the structure and conditions in a thermal conducting process, very useful and advantageous in novel material design.

\section{Numerical methods}

As stated above that closed theoretical solutions of the temperature field can only be obtained for such ideal structures as uniform, series or parallel distribution of each non-interactive phase. For most real complex media, the temperature field and thermal responses should be obtained numerically. 
The lattice Boltzmann method (LBM) is intrinsically a mesoscopic approach based on the evolution of statistical distribution on the lattices, and has achieved considerable success in simulating fluid flows and associated transport phenomena ${ }^{[29-32]}$, for especially its easy implementations of multiple inter-particle interactions and dealing with complex boundary conditions [33-35]. Herein, we used a LBM algorithm ${ }^{[16,20]}$ for calculating the temperature field with conjugate heat transfer in porous media, which was validated by comparing the numerical results with the existing theoretical solutions and experimental data, and adapt it for effective thermal conductivity predictions. The main idea of this LBM algorithm is introduced as follows.

For heat conduction in porous media with no heat source and no phase change, the temperature evolution equation for each phase is generally written as

$$
g_{\alpha}\left(r+e_{\alpha} \delta_{t}, t+\delta_{t}\right)-g_{\alpha}(r, t),
$$

$$
=-\frac{1}{\tau_{n}}\left[g_{\alpha}(r, t)-g_{\alpha}^{e q}(r, t)\right]
$$

where the equilibrium distribution of the evolution variable, $g_{\alpha}$, for the two-dimensional nine-speed (D2Q9) model, as shown in Fig. 1, is

$$
g_{\alpha}^{e q}= \begin{cases}0, & \alpha=0, \\ T / 6, & \alpha=1,2,3,4, \\ T / 12, & \alpha=5,6,7,8,\end{cases}
$$

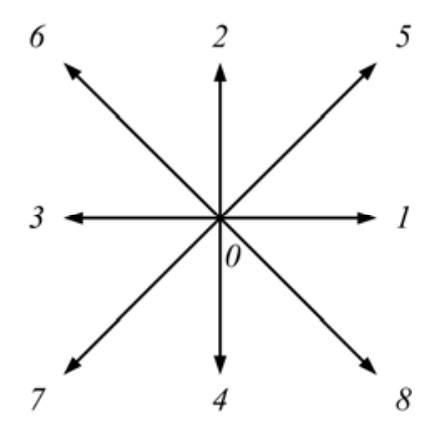

Fig. 1 Lattice structure of D2Q9

the microscopic velocity

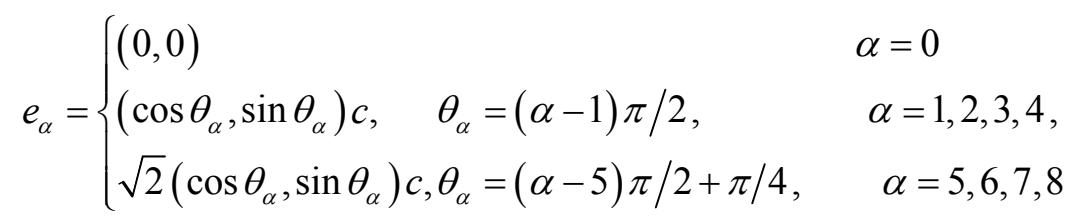

and the dimensionless relaxation time

$$
\tau_{n}=\frac{3}{2} \frac{k_{n}}{c^{2} \delta_{t}}+0.5
$$

where the subscript $n$ still represents the nth phase, $\delta_{t}$ the time step, $k$ the thermal conductivity, and $c$ the lattice speed defined as $\delta_{x}$ $/ \delta_{t}$, whose value can take any positive value theoretically only to insure $\tau$ values within (0.5, $2)^{[31]}$. The temperature and the heat flux are then calculated by

$$
\begin{gathered}
T=\sum_{\alpha} g_{\alpha}, \\
q=\left(\sum_{\alpha} e_{\alpha} g_{\alpha}\right) \frac{\tau_{n}-0.5}{\tau_{n}} .
\end{gathered}
$$

For the isothermal boundary treatment, the bounce-back rule of the non-equilibrium distribution proposed by Zou and $\mathrm{He}^{[37]}$ is used:

$$
g_{\alpha}-g_{\alpha}^{e q}=-\left(g_{\beta}-g_{\beta}^{e q}\right),
$$

where the subscripts $\alpha$ and $\beta$ represent two opposite directions respectively, and the equilibrium distribution can be calculated using the local boundary temperatures.

For the insulated boundary, a specular reflection boundary condition is implemented

$$
g_{\alpha}=g_{\gamma},
$$

where the subscripts $\alpha$ and $\gamma$ represent two directions satisfying specular reflection condition.

After the temperature field $T$ and $q$ are 
obtained by either theoretical or numerical calculation, the system ETC, $k_{\text {eff, }}$, can finally be determined as

$$
k_{\text {eff }}=\frac{L \int q d A}{\Delta T \int d A},
$$

where $q$ is the steady heat flux through the media cross-section area $d A$ between the temperature difference $\Delta T$ over a distance $L$. Meanwhile, the total and nondimensional entransy dissipation rate during heat conduction process can be obtained by using equation (5) and (7), respectively.

\section{Results and Discussion}

\subsection{Parallel and series models}

Since both the parallel mode and the series mode, as shown Fig.2, can be regarded as two basic structures of two-phase media, the irreversibility of heat conduction in these two simple porous media are first analyzed, and then the relation between the ETC and NER is discussed.

Assuming the thermal conductivities of each component are $k_{1}$ and $k_{2}\left(k_{1}<k_{2}\right)$, respectively, the temperature difference and the distance between the hot end and the cold end are $\Delta T$ and $L$, respectively, the volume of the heat conduction domain is $V_{0}$, the simple theoretical solutions give the effective thermal conductivities ETC as $\left(k_{1}+k_{2}\right) / 2$ for the parallel mode and $1 /\left(1 / 2 k_{1}+1 / 2 k_{2}\right)$ for the series mode..

Meanwhile, by selecting $k_{1}$ as the reference heat conductivity, the nondimensional entransy dissipation rate NER for the parallel mode and the series mode are $\left(k_{1}+k_{2}\right) / 2 k_{1}$ and $1 /(1 / 2+$ $\left.k_{1} / 2 k_{2}\right)$, i.e., both $1 / k_{1}$ timing its corresponding ETC. This result illustrates that the ETC not only influences the rate of heat transfer in a porous medium for given boundary conditions, but proportionates to the NER, i.e. the reduction of the heat transfer capacity of the medium during the heat conduction process. That is to say, ETC reflects the irreversibility when heat transfers through the porous media, just as the viscosity reflects the irreversibility when the fluid flows.

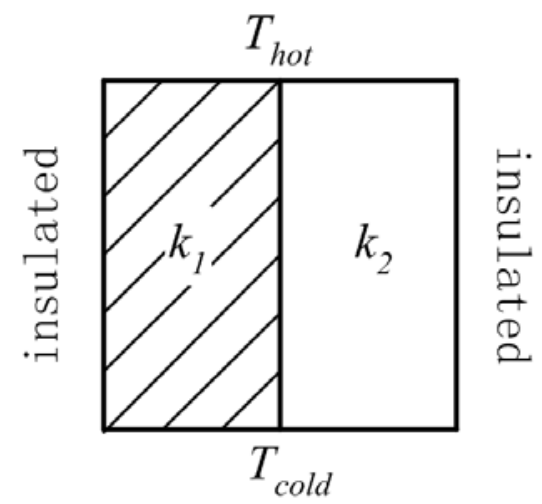

(a) Parallel mode

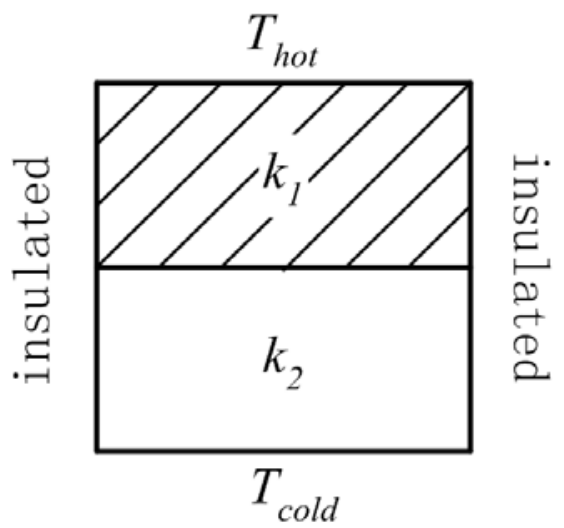

(b) Series mode

Fig. 2 Two basic structures of porous media

\subsection{Regular Pore/particle distribution}

Several researchers have reported that decreasing the average pore/particle sizes increases the effective thermal conductivities of porous media for given components and porosities of the media utilizing the experimental ${ }^{[38-39]}$ or/and numerical ${ }^{[16]}$ methods, which is an important phenomenon for porous media designs, yet still poorly understood.

In this section, the pore/particle size effect to the ETC of porous media are discussed based on the concept of entransy dissipation. Consider a 2-D heat conduction structure as shown in Fig. 3. The square heat conduction domain is divided into $2 n \times 2 n$ parts symmetrically $(n=1,2,3, \cdots)$. Two components with the thermal conductivities of $k_{1}=1 \mathrm{~W} / \mathrm{mK}$ and $k_{2}=100 \mathrm{~W} / \mathrm{mK}$ placed in every two separate parts, respectively, and the 
volume fraction of each component is 0.5 . The top and bottom boundaries are isothermal with the nondimensional temperatures of 1 and 0 , respectively, while the left and right boundaries are insulated.

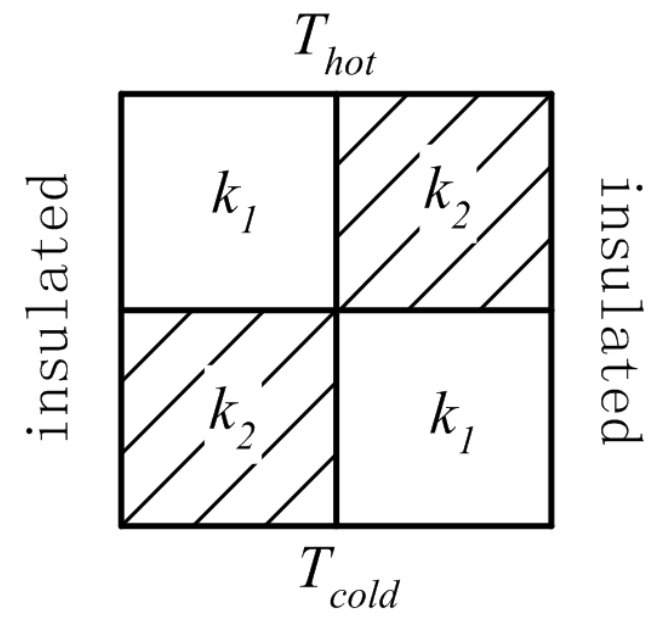

(a) $n=1$

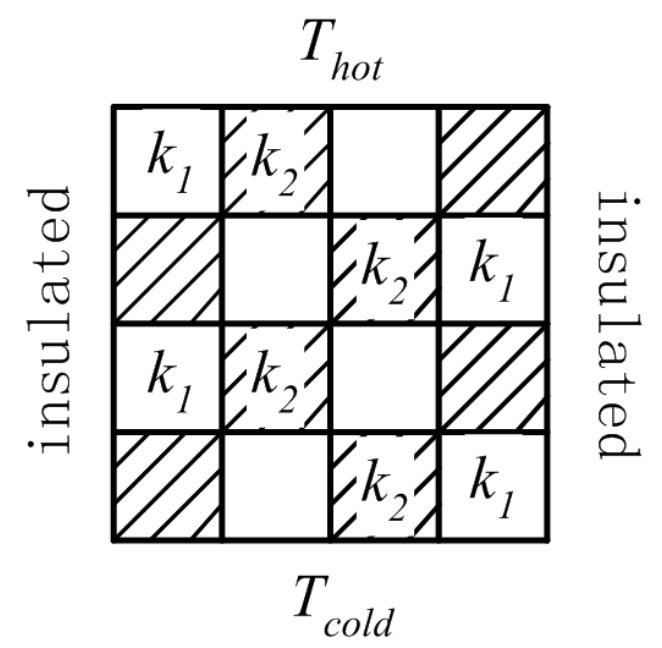

(b) $n=2$

Fig. 3 Porous media with uniform particle distribution

The predicted ETCs and NERs for different values of $n$ are shown in Fig. 4, indicating that both ETC and NER increase with the value of $n$. Since the particle volume is inversely proportional to the value of $n$, the result means that both the ETC and the NER of porous media during a heat conduction process decrease monotonically with the particle average size. From the values of NER, it is concluded that a larger $n$ value leads to a greater ETC and as $n$ changes, the range for $1 /\left(1 / 2 k_{1}+1 / 2 k_{2}\right)<$ ETC $<\left(k_{1}+k_{2}\right) / 2$.

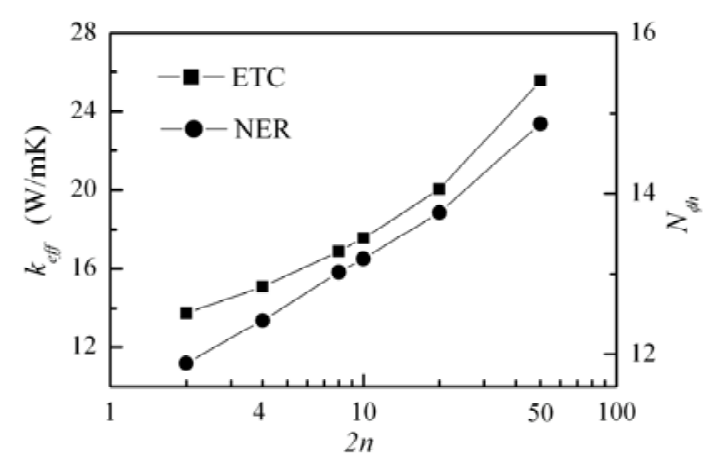

Fig. 4 ETC and NER versus value of $n$

In order to further explore the phenomenon, Fig.5 shows the temperature contours in the media for the structures of $n=1,2$ and 25. As shown in Fig. 5(a), the magnitude of the temperature gradient in most of the heat conduction domain is relatively small, while it is quite large near the common contact point, or the center, of each block formed by the neighboring four particles, resulting in a large heat flux in this point. That is to say, the area nearby the contact point is a heat bridge in the entire heat conduction domain. Based on the conventional heat transfer enhancement viewpoint, increasing the heat bridges (contact points) may effectively enhance heat transfer. As shown in Fig. 3(b), there are nine heat bridges (contact points), where the magnitude of the temperature gradient is smaller compared to the result of $n=1$ as shown in Figs. 5(a) and (b), thus the thermal resistance deceased or the ETC of porous media increased. By continuing to increase the heat bridges with increasing the value of $n$, the temperature gradient near the contact points further decreased or the ETC significantly increased. As shown in Fig. 5(c), when the value of $n$ is 25 , the temperature contours is relative smooth, close to those in homogeneous materials with the smallest temperature gradient. For this case, it seems as there exists a heat bridge network in the heat 
conduction domain. Thus, compared to the structure of $n=1$, the ETC increased by $90 \%$ when $n=25$.

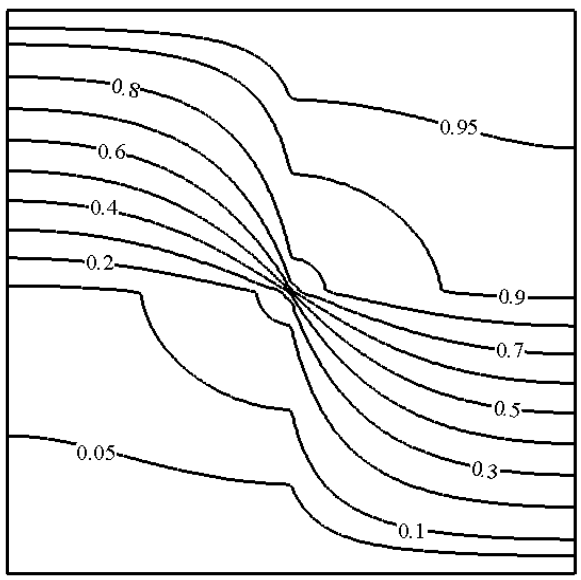

(a) $n=1$

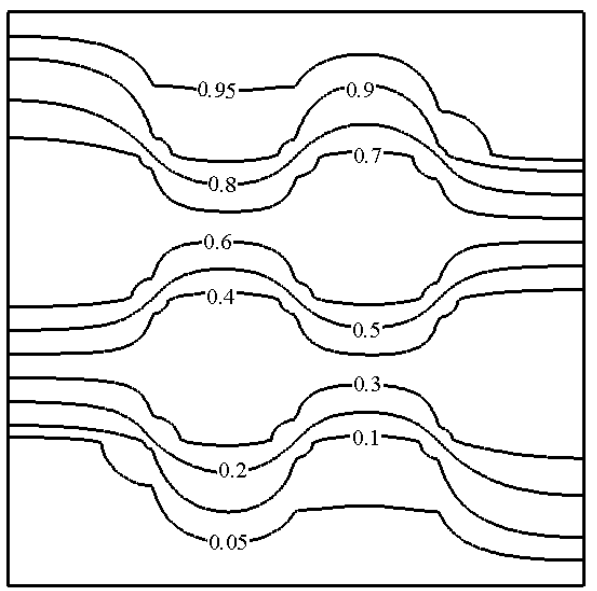

(b) $n=2$

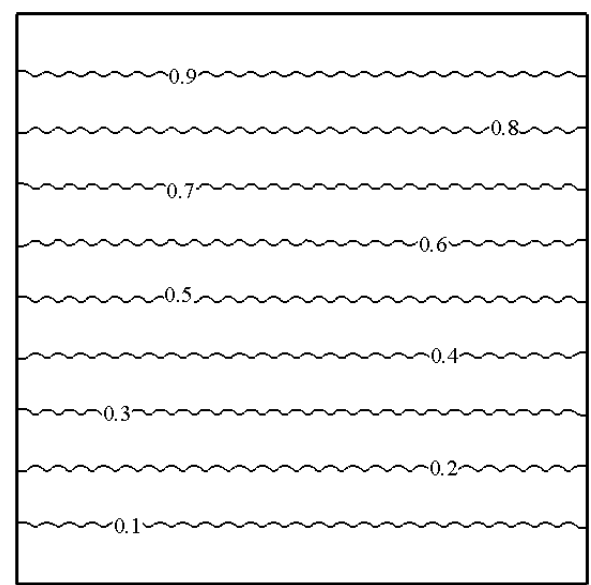

(c) $n=25$

Fig. 5 Temperature contours for different values of $n$

Furthermore, in order to find out the essential reason why decreasing the particle size may increase the ETC of porous media, Figs.6(a) and 6(b) show the local NER contours in the porous media for the structures of $n=1$ and 2, respectively. The greater the temperature gradient near the contact point, the larger the local entransy dissipation at this point than those in the other areas. Comparing Figs. 6(a) and 6(b), we found that increasing the contact point may decrease the local entransy dissipation nearby the contact point, which in turn increases the uniformity of the local entransy dissipation in the entire heat conduction area. For prescribed temperature boundary conditions, the integral of the local temperature gradient along the heat flow direction is constant, equaling to the temperature difference between the hot end and the cold end. Meanwhile, the total entransy dissipation rate is the integral of the local entransy dissipation rate, which is proportional to the square of the magnitude of local temperature gradient. Thus a uniform local temperature gradient along the heat flow direction will result in a uniform local NER distribution, consequently a large total NER or ETC of the media. 


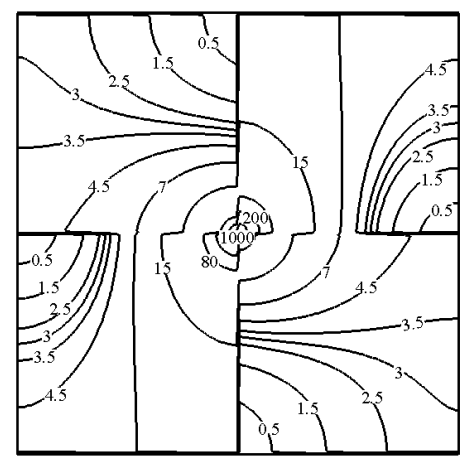

(a) $n=1$

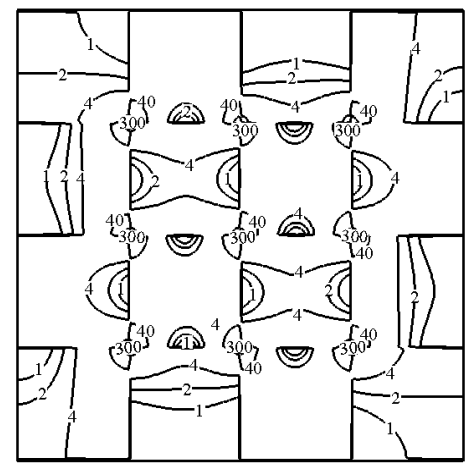

(b) $n=2$

Fig. 6 Local entransy dissipation rate contours for different values of $n$

\subsection{Random Pore/particle distribution}

In practical application, it is nearly impossible to distribute the pore/particle uniformly with the same size, shape and orientation. Therefore, it is necessary to validate the relation between the NER and ETC in the porous media with random pore/particle distribution.

Herein, the QSGS method, proposed by Wang et al. ${ }^{[16]}$, was utilized to generate the random microstructure of porous media. The average pore/particle size was determined by the values of core distribution possibility, $c_{d}$. A greater value of $c_{d}$ leads to a smaller average size of pores/particles for a certain porosity $\varepsilon$. Fig. 7 demonstrates two thus generated structures with the volume fraction of each component $\varepsilon=0.5$, where (a) has a hundred times higher $c_{d}$ value than (b), leading to a much smaller average particle size.

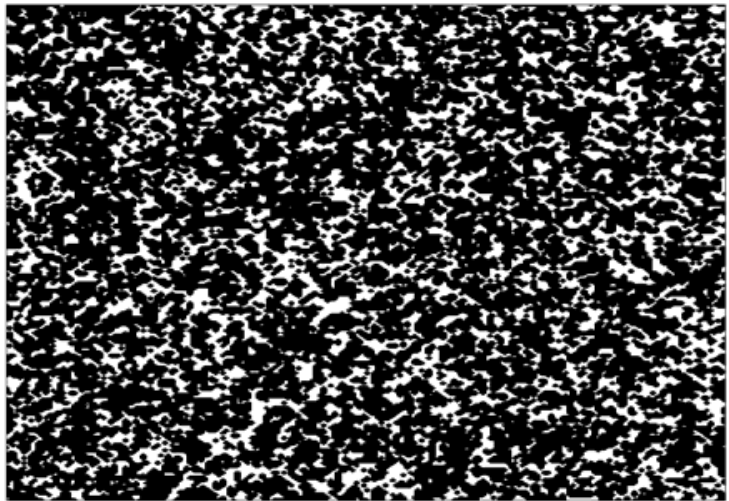

(a) $c_{d}=0.3 \varepsilon$

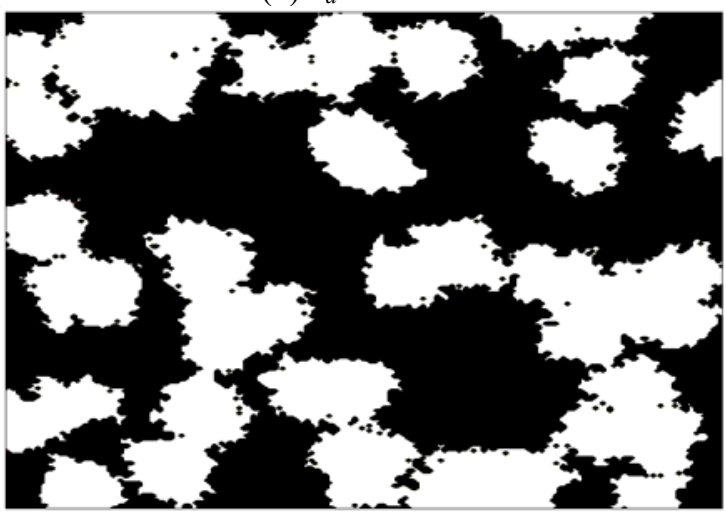

(b) $c_{d}=0.003 \varepsilon$

Fig. 7 Structures for different values of $c_{d}$ at a same porosity $\varepsilon=0.5$. The dark is component 1 with $k_{1}=1 \mathrm{~W} / \mathrm{mK}$ and the white is component 2 with $k_{2}=100 \mathrm{~W} / \mathrm{mK}$

After the porous structures are generated for different values of $c_{d}$, the relation between the ETC of porous media and the NER during heat conduction processes with various particle sizes is investigated. The thermal conductivities of the components are also $1 \mathrm{~W} / \mathrm{mK}$ and $100 \mathrm{~W} / \mathrm{mK}$, respectively. The predicted ETCs and NERs for different values of $c_{d}$ are shown in Fig. 8, which indicates that the values of both ETC and NER increase with the core distribution possibility, $c_{d}$, i.e., both the ETC of porous media and the NER during heat conduction process decrease monotonically with the particle average size. 


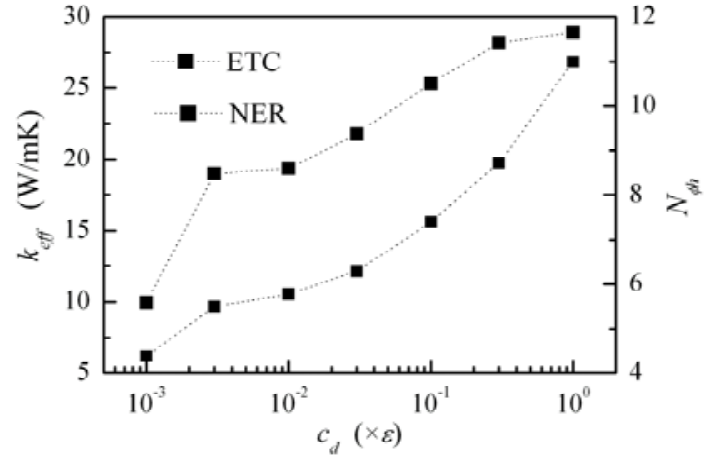

Fig. 8 ETC and NER versus value of $c_{d}$ at $\varepsilon=$ 0.5

\section{Conclusions}

By analyzing the irreversibility of heat conduction in porous media and its relation to effective thermal conductivities, a nondimensional entransy dissipation rate (NER) has been developed in this study as an objective function in optimizing the thermal behaviors of a complex medium, or designing a material system with desired thermal performance for a given condition. Next different porous structures were generated, and the temperature fields in these structures were obtained, and finally both NER during heat conduction processes and the ETC of porous media were calculated. In other words, NER describes the efficiency of a thermal conduction process in a material, whereas ETC reflect the material resistance to thermal transfer.

Meanwhile, decreasing the particle size may increase the contact points, i.e. the heat bridges, decrease the temperature gradient nearby the contact points, and significantly increase the effective thermal conductivities of porous media. Essentially, for porous media with either uniform or random particle distribution, decreasing the particle size will result in a more uniform distribution of temperature gradient and local entransy dissipation rate along the heat flow direction, consequently lead to a large effective thermal conductivity.

\section{Acknowledgements}

The present work is supported by the National
Key Fundamental R\&D Program of China (Grant No. G2007CB206901).

\section{References}

[1] R. W. Zimmerman, Thermal conductivity of fluid-saturated rocks, J. Pet. Sci. Eng. 3 (1989) 219-227.

[2] Zimmerman R W. Compressibility of sandstones. Elsevier, New York, 1991.

[3] Quintard M, Todorovic M. Heat and mass transfer in porous media. Elsevier, New York, 1992.

[4] Verruijt A. Computational Geomechanics. Kluwer Academic, Boston, 1995.

[5] Ingham D B, Bejan A, Mamut E, Pop I. Emerging technologies and techniques in porous media. Kluwer Academic, London, 2003.

[6] Lu G Q, Zhao X S. Nanoporous materials science and engineering, chemical engineering series. Imperial College Press, London, 2004.

[7] Ingham D B, Pop I. Transport phenomena in porous media III. Elsevier, Oxford, UK, 2005.

[8] Sayari A, Jaroniec M. Nanoporous materials IV. Elsevier, New York, 2005.

[9] Thomson W J. Introduction to transport phenomena. Prentice-Hall, Inc., 1999.

[10] A. Bouguera, Prediction of effective thermal conductivity of moist wood concrete, J. Phys. D Appl. Phys. 32 (1999) 1407-1414.

[11] X. G. Liang, W. Qu, Effective thermal conductivity of gas-solid composite materials and the temperature difference effect at high temperature, Int. J. Heat Mass Trans. 42 (1999) 1885-1893.

[12] J. E. J. Staggs, Estimating the thermal conductivity of chars and porous residues using thermal resistor networks, Fire Safety J. 37 (2002) 107-119.

[13] M. Wang, N. Pan, Prediction of effective physical properties of complex multiphase materials. Mat. Sci. Eng. R., (2008), doi: 10.1016/j.mser.2008.07.001.

[14] L. Tacher, P. Perrochet, A. Parriaux, Generation of granular media, Transport porous Med. 26 (1997) 99-107.

[15] S. Mohanty, Effect of multiphase fluid saturation on the thermal conductivity of geologic media, J. Phys. D Appl. Phys. 30 (1997) L80-L84.

[16] M. Wang, J. K. Wang, N. Pan, S. Y. Chen, 
Mesoscopic predictions of the effective thermal conductivity for microscale random porous media, Phys. Rev. E. 75 (2007) 036702 .

[17] H. F. Zhang, X. S Ge, H. Ye, Effectiveness of the heat conduction reinforcement of particle filled composites. Model Simul. Mater. Sci. Eng. 13 (2005) 401-412.

[18] I. J. Turias, J. M. Gutierrez, P. L. Galindo, Modeling the effective thermal conductivity of an unidirectional composite by the use of artificial neural networks, Compos. Sci. Technol. 65 (2005) 609-619.

[19] S. Barta, P. Dieska, Effective thermal conductivity of particulate composite materials, Kovove mater. 40 (2002) 99-112.

[20] J. K. Wang, M. Wang, Z. X. Li, A lattice Boltzmann algorithm for fluid-solid conjugate heat transfer, Int. J. Therm. Sci. 46 (2007) 228-234.

[21] A. G. Fedorov, R. Viskanta, Three-dimensional conjugate heat transfer in the microchannel heat sink for electronic packaging, Int. J. Heat Mass Trans. 43 (2000) 399-415.

[22] A. Horvat, T. Catton, Numerical technique for modeling conjugate heat transfer in an electronic device heat sink, Int. J. Heat Mass Trans. 46 (2003) 2155-2168.

[23] X. Huai, W. Wang, Z. Li, Analysis of the effective thermal conductivity of fractal porous media, Appl. Therm. Phys. 27 (2007) 2815-2821.

[24] Z.Y. Guo, H.Y. Zhu, X.G. Liang, Entransy --a Physical Quantity Describing Heat Transfer Ability, Int. J. of Heat Mass Trans. 50 (2007) 2545-2556.

[25] J.A. Meng, X.G. Liang, Z.X. Li, Field Synergy Optimization and Enhanced Heat Transfer by Multi-longitudinal Vortices Flow in Tube, Int. J. Heat Mass Trans. 48 (2005) 3331-3337.

[26] Q. Chen, J. X. Ren, J. A. Meng, Field synergy equation for turbulent heat transfer and its application, Int. J. Heat Mass Tran. 50 (2007) 5334-5339.

[27] Q. Chen, J. X. Ren, Generalized thermal resistance for convective heat transfer and its relation to entransy dissipation, Chinese Sci. Bull. In press.

[28] J. Wu, X.G. Liang, The Application of entransy dissipation extremum principle in radiative heat transfer optimization, Sci. in Chin. E 51 (2008) 1306-1314.

[29] S. Y. Chen, G. D. Doolen, Lattice boltzmann method for fluid flows, Annu. Rev. Fluid Mech. 30 (1998) 329-364.

[30] M. Wang, and S. Chen. Electroosmosis in homogeneously charged micro- and nanoscale random porous media. J. Colloid Interface Sci. 3142007 264-273

[31] J. K. Wang, M. Wang, Z. X. Li, Lattice Poisson-Boltzmann simulations of electro-osmotic flows in microchannels, $J$. Colloid Interface Sci. 296 (2006) 729-736.

[32] M. Wang, J. K. Wang, Z. X. Li, Corrigendum to "Lattice Poisson-Boltzmann simulations of electro-osmotic flows in microchannels", $J$. Colloid Interface Sci. 300 (2006) 446.

[33] Q. J. Kang, D. X. Zhang, S. Y. Chen, Simulation of dissolution and precipitation in porous media, J. Geophys. Res. 108 (2003) 2505.

[34] Q. J. Kang, D. X. Zhang, P. C. Lichtner, I. N. Tsimpanogiannis, Lattice Boltzmann model for crystal growth from supersaturated solution, Geophys. Res. Lett. 31 (2004) L21604.

[35] M. Wang, J. K. Wang, S. Y. Chen, N. Pan, Electrokinetic pumping effects of charged porous media in microchannels using the lattice Poisson-Boltzmann method, J. Colloid Interface Sci. 304 (2006) 246-253.

[36] X. Chen, P. Han, A note on the solution of conjugate heat transfer problems using SIMPLE-like algorithms, Int. J. Heat Fluid Flow 21 (2000) 463-467.

[37] Q. S. Zou, X. Y. He, On pressure and velocity boundary conditions for the lattice Boltzmann BGK mode, Phys. Fluids 9 (1997) 1591-1598.

[38] K. Midttomme, E. Roaldset, The effect of grain size on thermal conductivity of quartz sands and silts, Pet. Geosci. 4 (1998) 165-172.

[39] J. Z. Liang, F. H. Li, Measurement of thermal conductivity of hollow glass-bead-filled polypropylene composites, Polym. Test. 25 (2006) 527-531. 